\title{
Validation of the Malay Version of the Hospital Anxiety and Depression Scale among Patients with Traumatic Brain Injury
}

Leong Abdullah $\mathrm{MFI}^{a}$, Sidi $\mathrm{H}^{b}, \mathrm{Ng} \mathrm{YP}^{c}$,

a Lifestyle Science Cluster, Advanced Medical and Dental Institute, Universiti Sains Malaysia, 13200 Bertam, Pulau Pinang, Malaysia

b Department of Psychiatry, Universiti Kebangsaan Malaysia, Jalan Yaacob Latif, Bandar Tun Razak, 56000 Cheras, Kuala Lumpur, Malaysia

c Department of Psychiatry, RCSI \& UCD Malaysia Campus, Jalan Sepoy Lines, 10450 George Town, Pulau Pinang, Malaysia

\section{ABSTRACT}

Introduction: Depression and anxiety are common complications associated with traumatic brain injury (TBI) patients but screening tools which are validated to assess these complications in the TBI population are scarce. This study investigated the validity of the Malay version of Hospital Anxiety and Depression Scale (HADS) to screen for depression and anxiety among Malaysian TBI patients. Materials and Methods: This cross -sectional study recruited $101 \mathrm{TBI}$ patients in which they were administered the Malay version of HADS and the Structured Clinical Interview for Diagnostic and Statistical Manual for Mental Disorder $4^{\text {th }}$ Edition (DSM-IV) for Axis I Disorders (SCID-I) (as comparison tool). Internal consistency (Cronbach's a) and concurrent validity (using receiver operating characteristics) of the Malay version of HADS were evaluated. Results: The total Malay version of HADS score and its anxiety subscale exhibited good internal consistency of 0.80 and 0.78 respectively, but the internal consistency of its depressive subscale was low at 0.57 . The depressive subscale of the Malay version of HADS exhibited high area under the curve (AUC) of 0.86 , specificity of $82 \%$, sensitivity of $76 \%$ and negative predictive value of $91 \%$ but its positive predictive value was $58 \%$, at cut-off point of $8 / 9$. While the anxiety subscale also demonstrated high AUC of 0.88 , specificity of $71 \%$, sensitivity of $93 \%$ and negative predictive value of $98 \%$ its positive predictive value was only $34 \%$. Conclusion: The Malay version of HADS is a valid screening tool for depression and anxiety among Malaysian TBI population.

KEYWORDS: HADS-Malay version, traumatic brain injury, validation

\section{INTRODUCTION}

Traumatic brain injury (TBI) is a condition in which any extracranial mechanical forces to the brain causes any of the following consequences: (a) any loss of memory of any event immediately before or after the trauma, (b) any period of loss of consciousness, (c) any alteration of mental state at the time of the trauma. ${ }^{1}$ Psychiatric complications resulted from TBI include depression, anxiety, apathy, mania, psychosis and aggressive behaviouror agitation. ${ }^{2}$ Among these psychiatric

Corresponding Author:

Dr Mohammad Farris Iman Leong Bin Abdullah

Lifestyle Science Cluster

Advanced Medical and Dental Institute

Universiti Sains Malaysia, Bertam,

13200 Kepala Batas, Pulau Pinang

Tel No: +604-5622482

Email: farris@usm.my complications, depression is the most common with its prevalence ranging from $6 \%$ to $77 \% .^{3,4,5}$ Additionally, $22 \%$ of patients with TBI have anxiety, in which the anxiety severity is greater among minor TBI patients compared to those with more severe TBI. ${ }^{6}$ Comorbid depression and anxiety disorders associated with traumatic brain injury are also frequently reported, in which $31 \%$ to $61 \%$ of those reported with depression also have comorbid anxiety disorders. ${ }^{7}$ In Malaysian TBI population, an unpublished study reported the prevalence of major depression to be at $20 \%$ while anxiety disorders are at 7.5\%.

Although there is a high prevalence of depressive and anxiety disorders among TBI patients, validated instruments for diagnosis and screening of depressive and anxiety disorders in TBI patients are scarce. The gold standard instrument for diagnosis of major 
depressive disorder in TBI patients is Structural Clinical Interview for DSM (SCID), particularly in patients with complicated presentation. ${ }^{4}$ Nevertheless, SCID is time consuming and impractical to be used in busy clinical settings as its administration requires intensive training. As a result, self-administered instrument is more appropriate in clinical settings as it is easy to use, do not require extensive training and is time-effective. ${ }^{8}$

Among self-administered instruments used to assess depression includes Beck Depression Inventory (BDI), Depression, Anxiety and Stress Scale (DASS), Patient Health Questionaire-9 (PHQ-9), and Hospital Anxiety and Depression Scale (HADS) while those used for assessing anxiety are DASS, Beck Anxiety Inventory (BAI), and HADS. Only HADS and DASS were designed to assess both depression and anxiety but when considering which tool to be more suitable for TBI patients, HADS is preferred as it focus on the psychological rather than physical symptoms of depression which the latter could present as symptoms of $\mathrm{TBI}$ as well. To date, only 3 studies have been conducted to assess the psychometric properties of HADS in TBI patients, in which two studies evaluated the original English version of HADS while one study examined the Arabic version of HADS. While the English versions of the HADS are found to be a reliable and valid tool to screen for depression and anxiety among the TBI populations, the Arabic version of the HADS is ineffective as a screening tool for depression in the TBI cohort. ${ }^{8,9,10}$ Despite the fact that HADS has been translated into Malay and validated as a screening tool for depression and anxiety in Malaysian breast cancer caretakers, ${ }^{11}$ HADS (Malay) has not been validated for use in TBI patients. Hence, the goal of this study is to evaluate the psychometric properties of HADS (Malay) among Malaysian TBI patients.

(a) Based on sensitivity:

$$
\mathrm{TP}+\mathrm{FN}=\mathrm{z}^{2} \times \frac{[\mathrm{Sn}(1-\mathrm{Sn})]}{\mathrm{W}^{2}} \text { and } \mathrm{N}(\mathrm{Sn})=\frac{\mathrm{TP}+\mathrm{FN}}{\mathrm{P}}
$$

and (b) Based on specificity:

$\mathrm{FP}+\mathrm{TN}=\mathrm{z}^{2} x \frac{[\text { Spec }(1-\mathrm{spec})]}{\mathrm{W}^{2}}$ and $\mathrm{N}(\mathrm{Spec})=\frac{\mathrm{FR}+\mathrm{TN}}{1-\mathrm{P}}$

where: $\mathrm{TP}=$ true positive

$\mathrm{TN}=$ true negative

$\mathrm{FP}=$ False positive

$\mathrm{FN}=$ False negative

$\mathrm{Sn}=$ sensitivity

Spec $=$ specificity

$z=1.96$ for confidence interval of $95 \%$

$\mathrm{W}=$ level of precision $\mathrm{P}=$ prevalence
Based on the study of Dahm et al. (2013), sensitivity and specificity of the HADS were 0.9 and 0.7 respectively, level of precision was set at $10 \%$ and the estimated prevalence of depression and anxiety in TBI patients was at $30 \% .{ }^{6,7}$ Hence, we concluded the sample size required was 110 patients (after adding $10 \%$ drop out rate).

The Neurosurgical Clinic of Hospital Pulau Pinang is a tertiary referral centre for traumatic brain injury in the Northern region of Peninsular Malaysia. This study recruited patients with traumatic brain injury (TBI) from Neurosurgery Clinic of Hospital Pulau Pinang, Malaysia over a period of 7 months by convenient sampling. All patients with traumatic brain injury who attended the Neurosurgical Clinic of Hospital Pulau Pinang were approached by the research team and screened for inclusion and exclusion criteria. The inclusion criteria includes: (a) those with mild to severe traumatic brain injury within 3 months prior to their presentation to the clinic, (b) aged 18 years and above, (c) proficient in the Malay language to complete the HADS and SCID-I, (d) those with Kanofsky performance scale score of at least 70 (the Kanofsky performance scale is the gold standard instrument used to measure the performance level or performance of activity of daily living. The score of 70 and above denotes that the patient is able to at least care for himself or herself and perform basic activity of daily living. This inclusion criterion is selected as we would like to recruit patients who are able to answer the questionnaires on their own without any significant assistance from others), ${ }^{13}$ and (e) those who are able to provide signed informed consent. The exclusion criteria are: (a) those with history of pre-existing psychiatric illnesses before sustaining traumatic brain injury, (b) those with past history of traumatic brain injury and other neurological disorders, and (c) those who are aggressive, psychotic, physically ill and with significant cognitive impairment which affect their comprehension of the consent form or being cooperative in the study. Those who fulfilled all inclusion criteria without any exclusion criteria were made to sign informed consent prior to enrolment in the study.

\section{Measures}

The respondents were administered with the semistructured questionnaire which collects data on socio -demographic characteristics (such as age, gender, 
ethnicity, religion, education level, employment status, and marital status) and clinical characteristics (such as nature of head injury, duration of TBI, area of brain injury, and cause/ mechanism of injury). The respondents were also administered Hospital Anxiety and Depression Scale (HADS) (Malay) and Structured Clinical Interview for DSM IV

Axis I Disorders (SCID-I)-Research Version.

The Malay version of the Hospital Anxiety and Depression Scale (HADS)

The Hospital Anxiety and Depression Scale is a selfadministered questionnaire that assesses patients' level of anxiety and depression. It comprised of fourteen items, in which seven items assess depression and another seven items assess anxiety. It is suitable for use in assessing level of depression and anxiety in patients with medical and surgical illnesses such as those with traumatic head injury as HADS focus on the psychological rather than on physical symptoms in which the latter may be present in both traumatic head injury, and depression and anxiety. Each item scores from 0 to 3 , and the range for both depressive and anxiety items is from 0 to $21 .{ }^{14}$ Validation of the English version of demonstrated good to excellent internal consistencies (Cronbach a) for total HADS, HADS depression and anxiety subscale of $0.94,0.88$ and 0.92 respectively. ${ }^{8}$ The Malay version of HADS has appropriate psychometric properties. It has Cronbach's a of 0.88 for anxiety subscale and 0.79 for depression subscale. Its sensitivity was measured by the mean differences and Effect Size Index (ESI), in which mean difference was 1.5 with ESI of 0.21 for anxiety and mean differences was 1.26 with ESI of 0.19 for depression. ${ }^{11}$ We utilised this Malay version of the HADS for our study.

Structured Clinical Interview for DSM-IV Axis I Disorders (SCID-I) -Research Version

SCID is a semi-structured interview questionnaire which used Diagnostic and Statistical Manual for Psychiatric Disorder (DSM) diagnostic criteria to accurately diagnose psychiatric disorder. It is a semi -structured interview questionnaire which is administered by Psychiatrist or Clinical Psychologist or personnel who are extensively trained and familiar with the use of open ended questions in interviewing psychiatric patients. SCID is broken down into different diagnostic categories. Each category starts with an entry question which allowed the interviewer to skip associated questions if the entry question criterion is not met. Diagnosis of a psychiatric disorder is made according to the diagnostic algorithm for that particular disorder in SCID. SCID has good reliability and validity. It was demonstrated to have high kappa values of 0.7 to $1.00^{15}$ The licence for use of SCID-I Research Version was purchased from Biometrics Research at Columbia University. In this study, SCID-I was used as a gold standard comparison for the Malay version of HADS.

\section{Data analysis}

All data was analysed using SPSS version 22 . Descriptive statistics for socio-demographic and clinical characteristics of patients were computed. Internal consistency was assessed with Cronbach's a. Concurrent validity was computed using receiver operating characteristic (ROC) curve analysis, in which sensitivity' (true positive rate) was plotted against '1 - Specificity' (false positive rate), where SCID-I was used as the gold standard comparison. The concurrent validity was determined by calculating the area under the ROC curve (AUC). The receiver operating characteristics (ROC) curve was computed with Youden index $(\mathrm{J})$ to determine the optimal cut-off scores of the Malay version of HADS. By using the formula 'Sensitivity + Specificity -1', vertical distance of each cut-off scores were calculated from the receiver operating characteristic curve, and the score with the greatest distance is identified as the Youden index. ${ }^{16}$ The corresponding cut-off point is determined and taken as the optimal threshold value of the test, based on Youden index. ${ }^{17}$ The positive predictive values (PPV) and negative predictive values (NPV) associated with the cut-off scores were also computed.

\section{RESULTS}

Socio-demographic and clinical characteristics of respondents

There was $110 \mathrm{TBI}$ patients who met the inclusion criteria of the study but 9 patients refused to participate. In total this study recruited 101 patients. The socio-demographic and clinical characteristics of the respondents were summarized in Table I. 
Table I. Socio-demographic and clinical characteristics of respondents

\begin{tabular}{|c|c|c|}
\hline Variables & $\begin{array}{l}\text { Number of } \\
\text { subjects (n) }\end{array}$ & $\begin{array}{l}\text { Percentage } \\
\text { (\%) }\end{array}$ \\
\hline $\begin{array}{l}\text { Mean age } \\
\text { (standard deviation) } \\
\text { Gender: }\end{array}$ & $37.1^{\#}$ & $16.5^{ \pm}$ \\
\hline Male & 85 & 84.2 \\
\hline Female & 16 & 15.8 \\
\hline \multicolumn{3}{|l|}{ Ethnicity: } \\
\hline Malay & 49 & 48.5 \\
\hline Chinese & 32 & 31.7 \\
\hline Indian & 19 & 18.8 \\
\hline Others & 1 & 1.0 \\
\hline \multicolumn{3}{|l|}{ Religion: } \\
\hline Muslim & 50 & 49.5 \\
\hline Buddhist & 24 & 23.8 \\
\hline Hindu & 17 & 16.8 \\
\hline Christian & 4 & 4.0 \\
\hline Others & 6 & 5.9 \\
\hline \multicolumn{3}{|l|}{ Education status: } \\
\hline Primary & 18 & 17.8 \\
\hline Secondary & 74 & 73.3 \\
\hline Tertiary & 9 & 8.9 \\
\hline \multicolumn{3}{|l|}{ Employment status: } \\
\hline Employed & 43 & 42.6 \\
\hline Unemployed & 53 & 52.5 \\
\hline Studying & 5 & 5.0 \\
\hline \multicolumn{3}{|l|}{ Marital status: } \\
\hline Single & 54 & 53.5 \\
\hline Married & 46 & 45.5 \\
\hline Divorced & 1 & 1.0 \\
\hline \multicolumn{3}{|l|}{ Nature of head injury: } \\
\hline Extradural haemorrhage & 16 & 15.8 \\
\hline Subdural haemorrhage & 26 & 25.7 \\
\hline Intracerebral haemorrhage & 25 & 24.8 \\
\hline Contusion & 11 & 10.9 \\
\hline Mixed/multiple types & 21 & 20.8 \\
\hline \multicolumn{3}{|l|}{ Mean duration of TBI in } \\
\hline (Standard deviation) & 10.3 & 19.1 \\
\hline \multicolumn{3}{|l|}{ Mechanism of trauma: } \\
\hline MVA & 91 & 90.0 \\
\hline Assault & 5 & 5.0 \\
\hline Fall & 3 & 3.0 \\
\hline Others & 2 & 2.0 \\
\hline \multicolumn{3}{|l|}{ Area of brain injury: } \\
\hline Right hemisphere & 38 & 37.6 \\
\hline Left hemisphere & 31 & 30.7 \\
\hline Bilateral & 20 & 19.8 \\
\hline Midline & 9 & 8.9 \\
\hline
\end{tabular}

\# = mean, ${ }^{ \pm}=$standard deviation

The mean age of the respondents was 37.1 years $( \pm 16.5)$. Majority of the respondents were male $(84.2 \%, n=85)$ while almost half of the respondents were Malay $(48.5 \%, \mathrm{n}=49)$ followed by Chinese $(31.7 \%, n=32)$ and Indian (18.8\%, $n=19)$. Seven tenth of the respondents had secondary education $(73.3 \%$, $\mathrm{n}=74)$, about half of them were unemployed $(52.5 \%$, $n=53)$ and about half were also single $(53.5 \%, n=$ 54). The clinical characteristics of the respondents revealed that the mean duration of TBI was 16.3 months $( \pm 19.3)$ while majority of them sustained TBI from motor vehicle accidents $(90 \%, n=91)$. Almost equal proportions of respondents were diagnosed with subdural haemorrhage (25.7\%, $\mathrm{n}=26)$, intracerebral haemorrhage $(24.8 \%, \mathrm{n}=25)$ and mixed type of injuries $(20.8 \%, n=21)$ while almost equal proportions of respondents had brain injury over the right hemisphere $(37.6 \%, n=38)$ and left hemisphere $(30.7 \%, n=31)$, followed by bilateral injury $(19.8 \%, n=20)$ and midline injury $(8.9 \%, n=9)$.

Reliability of the HADS (Malay) for diagnosis of depression and anxiety in TBI patients

With reference to the reliability of HADS (Malay) in assessing depression and anxiety in TBI patients, the internal consistency (Cronbach's a) of the HADS (Malay) total score was 0.81 . The internal consistency of the depressive subscale of the HADS (Malay) (Cronbach's a) was 0.57 and that of anxiety subscale was 0.78 .

Concurrent validity of the HADS (Malay) for diagnosis of depression and anxiety in TBI patients

The concurrent validity measures were summarized in Table II. In the assessment of concurrent validity, the ROC curve for the depression subscale of the HADS (Malay) was plotted against SCID-I diagnosis of depression (Figure 1) while the ROC curve for the anxiety subscale of the HADS (Malay) was plotted against SCID-I diagnosis of anxiety disorders (Figure 2 ). The area under the curve (AUC) of the ROC curve of the depressive subscale of the Malay version of HADS was high for depressive disorders which was $0.86 \pm 0.04(0.78-0.95)$ while the area under the curve (AUC) of the ROC curve of the anxiety subscale of the Malay version of HADS was also high for anxiety disorders which was $0.88 \pm 0.05(0.77-0.98)$.

Analysis of ROC of the Youden indexes for each curve $\left(J_{D}\right)$ for the depressive subscale of the Malay version of HADS and the Youden indexes for each curve $\left(\mathrm{J}_{\mathrm{A}}\right)$ for the anxiety subscale of the Malay version of HADS against SCID-I depressive disorders and anxiety disorders respectively revealed the optimal cut-off score for the depressive subscale for the Malay version of HADS to screen for major depressive disorder was $8 / 9$ while the anxiety subscale for the Malay version of HADS to screen for generalized anxiety disorder was $6 / 7$.

We also analysed for specificity, sensitivity, positive predictive value, negative predictive value, positive likelihood ratio and negative likelihood ratio. The depressive subscale of the Malay version of HADS demonstrated specificity of $76 \%$, sensitivity of $82 \%$, 
Table II. Summary of validity measures of the HADS (Malay) against the SCID-I in screening for depression and anxiety in TBI patients

\begin{tabular}{|c|c|c|c|c|c|c|c|c|c|c|}
\hline $\begin{array}{l}\text { HADS } \\
\text { subscale }\end{array}$ & $\begin{array}{l}\text { Cronbach's } \\
\text { a }\end{array}$ & $\mathrm{AUC}^{\#}$ & $\begin{array}{l}\mathrm{AUC} \\
\mathrm{Cl}^{\#}\end{array}$ & $\begin{array}{l}\text { Cut- } \\
\text { off }\end{array}$ & $\begin{array}{l}\text { Specificity } \\
\text { (\%) }\end{array}$ & $\begin{array}{l}\text { Sensitivity } \\
\text { (\%) }\end{array}$ & $\begin{array}{l}\text { PPV }^{\#} \\
\text { (\%) }\end{array}$ & $\begin{array}{l}\text { NPV }^{\#} \\
(\%)\end{array}$ & $\mathrm{LR}+{ }^{\#}$ & LR- ${ }^{\#}$ \\
\hline $\begin{array}{l}\text { Depressive } \\
\text { subscale of } \\
\text { the HADS } \\
\text { (Malay) }\end{array}$ & 0.57 & 0.86 & $\begin{array}{l}0.78- \\
0.95\end{array}$ & $8 / 9$ & 76 & 82 & 58 & 91 & 4.2 & 0.3 \\
\hline $\begin{array}{l}\text { Anxiety sub- } \\
\text { scale of the } \\
\text { HADS } \\
\text { (Malay) }\end{array}$ & 0.78 & 0.88 & $\begin{array}{l}0.77- \\
0.98\end{array}$ & $6 / 7$ & 93 & 71 & 34 & 98 & 3.2 & 0.1 \\
\hline
\end{tabular}

\footnotetext{
${ }^{\#} \mathrm{AUC}=$ area under the curve, $\mathrm{AUC} \mathrm{Cl}=$ area under the curve $95 \%$ confidence intervals, $\mathrm{PPV}=\mathrm{positive} \mathrm{predictive} \mathrm{value,}$ $\mathrm{NPV}=$ negative predictive value, $\mathrm{LR}+=$ positive likelihood ratio,

LR-= negative likelihood ratio
}

positive predictive value of $58 \%$, negative predictive value of $91 \%$, positive likelihood ratio of 4.2 and negative likelihood ratio of 0.3 at cut-off score of $8 / 9$. The anxiety subscale of the Malay version of HADS demonstrated specificity of $71 \%$, sensitivity of $93 \%$, positive predictive value of $34 \%$, negative predictive value of $98 \%$, positive likelihood ratio of 3.2 and negative likelihood ratio of 0.1 at cut-off score of $6 / 7$.

\section{Discussion}

This study aimed to investigate the reliability and validity of the HADS (Malay) to screen for depression and anxiety in patients with TBI. The findings from assessment of internal consistency and concurrent validity indicated that the HADS (Malay) was reliable and valid for use in Malaysian TBI patients.

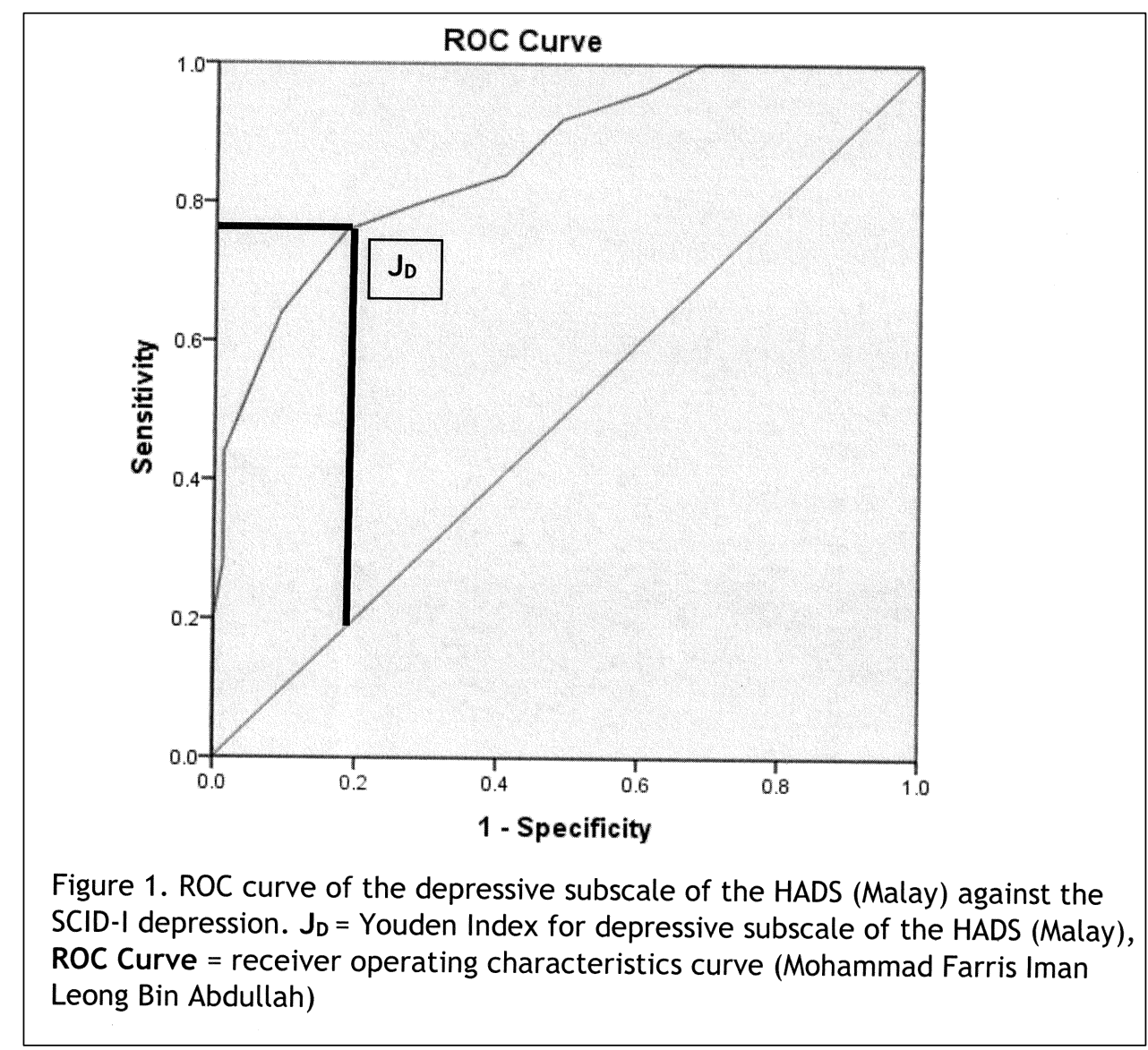




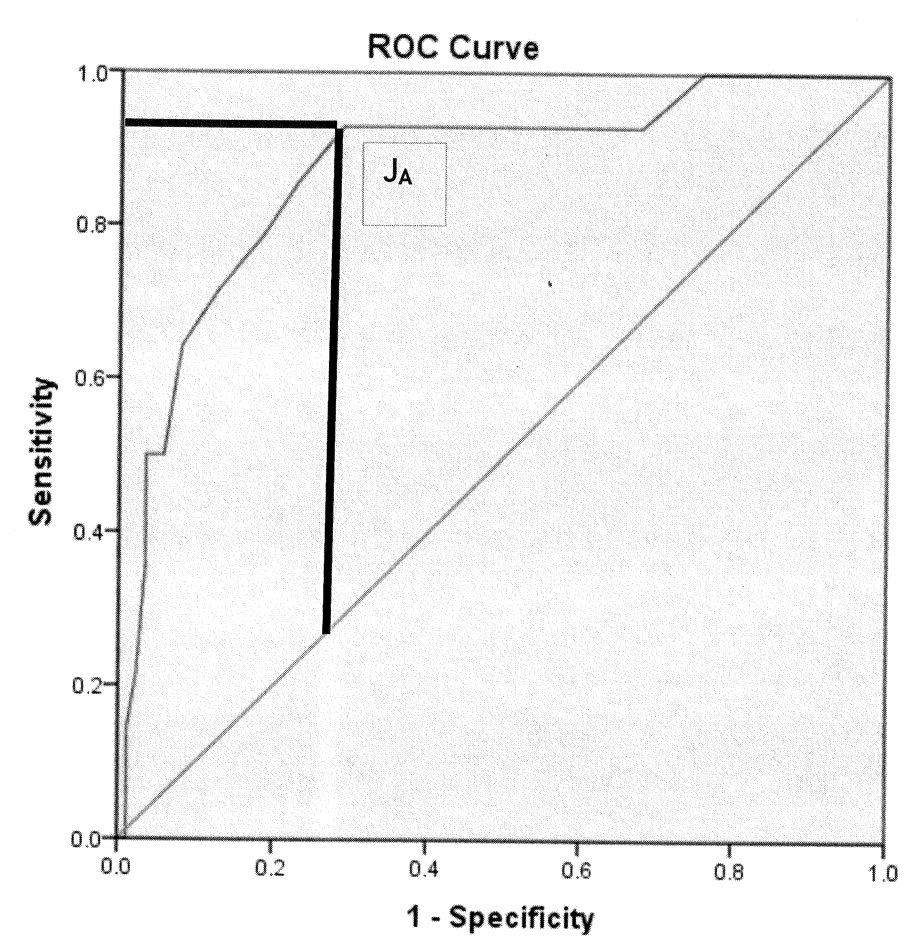

Figure 2. ROC curve of the anxiety subscale of the HADS (Malay) against the SCID-I anxiety disorders. $J_{A}=$ Youden Index for anxiety subscale of the HADS (Malay), ROC Curve $=$ receiver operating characteristics curve (Mohammad Farris Iman Leong Bin Abdullah)

The internal consistency of the HADS (Malay) total score in TBI patients was good according to interpretation of internal consistency by George and Mallory, 2003. It was comparable to that of the original English version of HADS for screening of depression and anxiety in English speaking TBI patients $^{8,10}$ and that of Malay version of HADS for screening depression and anxiety in non-TBI patients. ${ }^{11,18}$ The internal consistency of the anxiety subscale of the HADS (Malay) in this study was acceptable but the internal consistency of the depressive subscale of the HADS (Malay) was poor. ${ }^{19}$ We found that there was discrepancy of the wordings used in item 12 between the original English version and the Malay version of HADS where the sentence in the Malay version is relatively long, in which item 12 in the English version read as "I look forward with enjoyment to things" while item 12 in the Malay version read as "Dengan perasaan keseronokan, saya tidak sabar-sabar menunggu perkara-perkara yang akan terjadi". The sentence of item 12 in HADS (Malay) may have deviated from the original meaning of the English version HADS leading to low Cronbach's $a$ in the depressive subscale of HADS (Malay).

The Malay version of HADS used in this study was obtained from GL Assessment Ltd via MAPI Trust. Hence, the face, content and construct validities were not carried out as these would have been successfully validated in the Malaysian population. ${ }^{11}$ To determine the concurrent validity of the Malay version of HADS to screen for depression and anxiety in Malaysian TBI patients, we compared the Malay version of HADS with Structured Clinical Interview for DSM-IV Axis I Disorders (SCID-I) which is the gold standard instrument for diagnosis of depressive and anxiety disorders. The area under the curve (AUC) for the ROC of depressive subscale of the Malay version of HADS against SCID-I diagnosis of depressive disorders and the ROC of the anxiety subscale of the Malay version of HADS against SCID-I diagnosis of anxiety disorders were comparable to that of the AUC of ROC curves of the original English version of the HADS used for screening depression and anxiety in English-speaking TBI patients which were all above 0.8 and close to $1.0 .^{8,10}$

The Receiver Operating Characteristics (ROC) curve is a graphical plot of various values of sensitivity against ' 1 -specificity' and the area under the ROC curve (AUC) is an important measure of the intrinsic validity of a diagnostic tool. The maximum value of AUC is 1 which indicates that the tool under investigation is $100 \%$ accurate without any false negative and false positive tests. While the lowest acceptable value of AUC is 0.5 , in which any value below 0.5 indicates that the tool under investigation 
is not able to discriminate between normal and abnormal. The advantage of using ROC analysis is that it is not affected by prevalence of the disorder. ${ }^{20}$

The optimal cut-off point of the Malay version of HASDS in screening for depressive and anxiety disorders in TBI patients was determine by Youden index $(\mathrm{J})$ in our study. Youden index is identified as a point on the ROC curve which has the greatest vertical distance to the line of equality of the ROC curve. Youden index minimize false positive and false negative diagnoses and well supported by evidence for determining optimal cut-off point on ROC cruve. ${ }^{21}$ Our study demonstrated the optimal cut-off point of the depressive subscale of the Malay version of HADS in screening for depression in traumatic brain injury patients was $8 / 9$ while that of the anxiety subscale of the Malay version of HADS to screen for anxiety was $6 / 7$. Our findings were fairly close to that of the original English version of the HADS for screening depression and anxiety in TBI patients which manually defined cut-off point at $7 / 8 .^{8,10}$ A Saudi Arabia study on the validity of the HADS also supported our findings that the optimal cut-off point falls in the range of 7-9.22

The use of the Malay version of HADS to screen for depression and anxiety in traumatic brain injury patients demonstrated high sensitivity, specificity and negative predictive value (NPV) but a relatively low positive predictive value (PPV). The sensitivity, specificity and NPV of our findings were comparable to that of the two previous study on the validation of the original English version of the HADS for screening depression and anxiety in TBI patients where the sensitivity ranged from $62 \%-85 \%$, specificity ranged from $69 \%-92 \%$ and NPV ranged from $82 \%-90 \%$. However, PPV of the English version of the HADS is relatively higher which ranged from $57 \%-81 \%{ }^{8,10}$ We conclude that the Malay version of HADS was able to diagnose a large proportion of traumatic brain injury patients with depression and anxiety correctly, and it is able to exclude those without depression and anxiety. In fact, with NPV above $90 \%$ for all cut-off scores of its subscales, the HADS (Malay) is able to detect those who are without depression and anxiety among TBI patients as good as SCID-I. ${ }^{15}$ It should be noted that PPV is dependent on prevalence of the disease. Lower prevalence of disease lower the PPV of a screening instrument but increases the NPV of the instrument. ${ }^{23}$ Hence, a relatively low PPV in this study may be due to relatively lower prevalence of depression and anxiety in the TBI sample of this study. On the contrary, likelihood ratio (LR) is not dependent on prevalence of disease in a sample. Our findings showed the positive likelihood ratio $(L R+)$ of the depressive and anxiety subscales of the Malay version of HADS were above 1, implying that traumatic brain injury patients who scored above the optimal cut-off points were likely to have depressive and anxiety disorders. While the negative likelihood ratio (LR-) of the depressive and anxiety subscale of the Malay version of HADS were less than 0.3 (less than 1), indicating the Malay version of HADS was useful to exclude those without depressive and anxiety disorders.

There are two limitations of the study which must be noted. Firstly, the study sample was recruited from only one centre and therefore, it cannot be generalized to other TBI populations in Malaysia. Hence, a multicentric study would be more ideal. Finally, the internal consistency of the depressive subscale of the HADS (Malay) is debatable. Hence, the depressive subscale score of the HADS (Malay) used for assessment of depression in the Malaysian TBI population should be interpreted with caution. We recommend future study to re-look into the reliability of the translation of item 12 in the HADS (Malay) which may have resulted in relatively low internal consistency of the depressive subscale of the HADS.

\section{CONCLUSION}

HADS (Malay) was demonstrated to be a reliable and valid tool to screen for depression and anxiety among Malaysian patients with TBI. Due to the high prevalence of depression and anxiety among TBI patients, it would be pivotal to screen for these psychiatric complications in TBI patients and refer those who score above the cut-off scores for proper psychiatric treatment. Subsequently, this will help clinicians manage TBI patients in a holistic manner, treating not only the physical disabilities, but also the psychiatric complications which may arise. A validated Malay version of HADS would provide a useful screening tool to ensure Malaysian TBI patients are treated in a holistic manner.

\section{Conflict of interest}

None 


\section{REFERENCES}

1. Menon DK, Schwab K, Wright DW, et al. Position Statement: Definition of traumatic brain injury. Arch Phys Med Rehabil 2010; 91: 1637 40.

2. Ahmed S, Venigalla $H$, Mekala HM, et al. Traumatic brain injury and neuropsychiatric complications. Indian J Psychol Med 2017; 39:114-21.

3. Scholten AC, Haagsma JA, Cnossen MC, et al. Prevalence of and risk factors for anxiety and depressive disorders after traumatic brain injury: a systematic review. J. Neurotrauma 2016; 33:1969-94.

4. Seel RT, Macchiocchi S, Kreutzer JS. Clinical considerations for the diagnosis of major depression after moderate to severe TBI. J Head Trauma Rehabil 2010; 25:99-112.

5. Bombardier CH, Fann JR, Temkin NR, et al. Rates of major depressive disorder and clinical outcomes following traumatic brain injury. JAMA 2010; 303:1938-45.

6. van der Horn HJ, Spikman JM, Jacobs B, et al. Postconcussive complaints, anxiety, and depression related to vocational outcome in minor to severe traumatic brain injury. Arch Phys Med Rehabil 2013; 94:867-74.

7. Guillamondegui OD, Montgomery SA, Phibbs FT, et al. Traumatic brain injury and depression.

Comparative effectiveness review No. 25. Rockville, MD: Agency for Healthcare Research and Quality; 2011 Apr. AHRQ Publication No: 11 - EHC017-EF.

8. Whelan-Goodinson R, Ponsford J, Schonberger

M. Validity of the Hospital Anxiety and Depression

Scale to assess depression and anxiety following traumatic brain injury as compared with the Structured Clinical Interview for DSM-IV. J Affect Disord 2009; 114:94-102.

9. Al-Adawi S, Dorvlo ASS, Al-Naamani A, et al. The ineffectiveness of the Hospital Anxiety and Depression Scale for diagnosis in an Omani traumatic brain injured population. Brain Inj 2007; 21:385-93.

10. Dahm J, Wong D, Ponsford J. Validity of the Depression Anxiety Stress Scales in assessing depression and anxiety following traumatic brain injury. J Affect Disord 2013; 151:392-6.
11. Yusoff N, Low WY, Yip CH. Psychometric properties of the Malay Version of the Hospital Anxiety and Depression Scale: A study of husbands of breast cancer patients in Kuala Lumpur, Malaysia.

Asian Pac J Cancer Prev 2011; 12:915-7.

12. Buderer NMF. Statistical methodology: I. Incorporating the prevalence of disease into the sample size calculation for sensitivity and specificity. Academy of Emergency Medicine 1996; 3:895-900.

13. Greenberg MS. Handbook of Neurosurgery. $7^{\text {th }}$ ed. New York: Thieme Medical Publishers Inc, 2010.

14. Snaith RP, Zigmond AS. The Hospital Anxiety and Depression Scale Manual. GL Assessment Limited [Online]. Available at: www.gl-assessment.co.uk/products/hospitalanxiety-and-depression-scale-hads/ Accessed April 18, 2018.

15. First MB, Spitzer RL, Gibbon M, et al. Structured Clinical Interview for DSM-IV-TR Axis I Disorders, Research Version, Patient Edition. (SCID-I/P) New York: Biometrics Research, New York State Psychiatric Institute, 2002.

16. Unal I. Defining an optimal cut-point value in ROC analysis: an alternative approach. Comput Math Methods Med 2017; 2017:1-14.

17. Hajian-Tilaki K. Receiver operating characteristic (ROC) curve analysis for medical diagnostic test evaluation. Caspian J Intern Med 2013; 4:627-35.

18. Yong HW, Hashim Z, Jamil @ Osman Z. Reliability and validity of Hospital Anxiety and Depression Scale (HADS) on breast cancer survivors: Malaysia case study. APEOHJ 2016; 2:19-24.

19. George D, Mallery P. SPSS for Windows step by step: A simple guide and reference. 11.0 update. 4th ed. Boston: Allyn \& Bacon, 2003.

20. Kumar R, Indrayan A. Receiver Operating Characteristic (ROC) curve for medical researchers. Indian Pediatr 2011; 48:277-87.

21. Habibzadeh F, Habibzadeh $P$, Yadollahie $M$. Choice of criterion used in the receiver operating characteristic analysis. Indian J Med Res 2017; 145:571-2.

22. Al Aseri ZA, Suriya MO, Hassan HA, et al. Reliability and validity of the Hospital Anxiety and Depression Scale in an emergency department in Saudi Arabia: a cross-sectional 
observational

study. BMC Emerg Med 2015; 15:28.

23. Wong HB, Lim GH. Measures of diagnostic accuracy: Sensitivity, specificity, PPV and NPV.

PoSH 2011; 20:316-18. 
